\title{
DISEÑO SISMICO DE PUENTES: ESTADO DEL ARTE
}

\author{
William Lobo Quintero ${ }^{(l)}$
}

\begin{abstract}
RESUMEN
Se presenta en forma crítica el estado del conocimiento acerca del comportamiento de los puentes bajo excitaciones sísmicas y las medidas que los códigos modernos han venido implementando, con el objeto de adaptarlas para generar normativas propias de diseño sísmico de puentes en países que carecen de ellas. Se reafirma la necesidad de considerar los diseños basados en desempeño sísmico bajo estados límites, incorporando la variabilidad espacial y los movimientos verticales, favoreciendo la continuidad y el diseño conceptual, tomando en cuenta los efectos de focos cercanos, disponiendo de programas de análisis lineal y no lineal y modelos globales que incorporen el efecto de los estribos, la interacción suelo-estructura, el comportamiento de fundaciones, el aislamiento sísmico, la disipación de energía para mejorar el diseño de puentes nuevos y la evaluación, mantenimiento y rehabilitación de puentes existentes.
\end{abstract}

\begin{abstract}
The state of knowledge about the earthquake behavior of bridges and the modern codes measures are presented to generate bridge design standards in countries without them. It reaffirms the need to consider the performance based seismic design under limit states, to incorporate the spatial variability effects, the continuity of slabs and the conceptual design, that takes the near ground motions to support the linear and nonlinear analysis with sufficient and complete software and global models that include the connected abutments effects, the soil structure interaction, the behavior of foundations, the seismic isolation, the energy dissipation to improve the design of new bridges and the evaluation, maintenance and rehabilitation of existing bridges.
\end{abstract}

Artículo recibido el 16 de mayo de 2000 y aprobado para su publicación el 11 de octubre de 2000. Se aceptarán comentarios y/o discusiones al artículo hasta cinco meses después de su publicación.

(1) Profesor Titular, ULA, Mérida, Venezuela. E-mailwlobo@telcel.net.ve 


\section{ANTECEDENTES}

Una monografía hecha sobre 86 fuentes para el IV Congreso Venezolano de Sismología e Ingeniería Sísmica, permitió introducir en Venezuela las bases para la enseñanza, la investigación, la experimentación, la instrumentación y la redacción de unas Normas Sísmicas de Puentes (Lobo Quintero, 1984). En este trabajo, se recogieron las experiencias vividas con los terremotos, los requerimientos de análisis y diseño, el comportamiento real de pilas y estribos, el diseño de fundaciones, aisladores sísmicos y disipadores de energía, el comportamiento de los puentes pequeños y especiales, y la búsqueda de sistemas sísmicos adecuados. En los últimos quince años, los investigadores y los proyectistas han mejorado substancialmente el estado del arte y los códigos han cambiado en áreas tales como filosofía de diseño, criterios de desempeño, caracterización de sismos, efectos de sitio, análisis inelástico, procedimientos de diseño y sistemas protectores (Buckle, 1996).

Es necesario destacar el arte del diseño de puentes, para lograr soluciones racionales, regulares y simples de sistemas preferiblemente hiperestáticos, contínuos, livianos, bien construidos, detallados y fundamentados. El puente además de cumplir con una necesidad arquitectónica, salva una luz, y soporta pesadas cargas vibratorias. En los puentes son muy importantes las relaciones de estética a funcionalidad y al dar la impresión de ser simples de analizar; las respuestas sísmicas inadecuadas obligan a afinar la comprensión de su comportamiento (Calvi y Pinto, 1966). Hay aspectos a considerar cuidadosamente, como la diferencia en altura de las columnas, la presencia de partes adyacentes semiconectadas, la cantidad de apoyos no conectados, la diferencia entre la rigidez longitudinal y transversal, y el comportamiento no lineal de los pórticos transversales (Ramírez y Ayala, 1999a). La integridad estructural de un sistema se basa en la capacidad portante del terreno, el control de los desplazamientos laterales, la resistencia adecuada de los miembros, el detallado y confinado del concreto, y sobre todo la concepción del sistema resistente (Sauter, 1998).

La continuidad es importante, fácil y barata de obtener; pero, si las estructuras no son contínuas ni monolíticas, los tableros se deben conectar a apoyos o estribos para prevenir la apertura de juntas y evitar el colapso. El secreto de un buen diseño sismorresistente es balancear los niveles de daño aceptables con la economía para limitarlos (Roberts, 1996). Existe la evidencia de que en Venezuela y seguramente en el resto de América Latina, existen cientos de puentes no diseñados para cumplir normas sísmicas, y que requieren de programas de mantenimiento, evaluación y rehabilitación.

Una revisión de los puentes existentes en el Occidente de Venezuela, revela la falta de control de sobrecargas verticales, el peso adicional excesivo por repavimentaciones y el incumplimiento de normativas sísmicas actuales. Existen apoyos en mal estado, puentes sin losas de acceso, fundaciones muy superficiales, falta de conectores y de bloques de cortante, poco refuerzo de confinamiento, falta de detalles adecuados, carencia de ductilidad, rellenos sin control, asentamientos diferenciales, juntas destruidas, losas agrietadas, falta de simetría, pérdida de pernos, desprendimiento de arriostramientos, socavación de pilas y estribos, y poco mantenimiento. Por todo esto no se espera un comportamiento sísmico adecuado (Camargo, 1997). Un resumen de los daños típicos ocurridos comprende: caída de tramos, movimiento de tableros y estribos, falla estructural de columnas y pilas, licuación de suelos, inestabilidad de 
taludes, asentamiento de rellenos, incrementos de presión lateral, destrucción de apoyos y juntas (Lobo Quintero, 1997). La marcha de la investigación se ha sustentado sobre estas experiencias, tomando en cuenta bases teóricas, numéricas y experimentales, para revisar y adaptar continuamente las normativas vigentes. Este trabajo recopila los adelantos realizados con el fin de adaptarlos hacia el desarrollo de una ingeniería sísmica regional de puentes.

\section{DEMANDA SÍSMICA}

A pesar de que el uso de las historias de aceleraciones se va haciendo común, los códigos mantienen el uso de los espectros de diseño como la metodología más simple de estimar la demanda sísmica, donde el corte basal (V) puede obtenerse a través de expresiones del tipo:

$$
\mathrm{V}=\mathrm{C}_{1} \mathrm{C}_{2} \mathrm{C}_{3} \mathrm{~S}_{\mathrm{a}} \mathrm{W}
$$

donde $\mathbf{C}_{\mathbf{1}}$ es la relación entre desplazamientos elásticos e inelásticos, $\mathbf{C}_{\mathbf{2}}$ la degradación histerética de rigidez y de resistencia y $\mathbf{C}_{3}$ la modificación por efectos $\mathrm{P}-\Delta$ (FEMA, 1996); estos factores deben adaptarse a puentes. $\mathbf{S}_{\mathbf{a}}$ es la ordenada del espectro de pseudoaceleraciones entre la aceleración de la gravedad $\mathbf{g}$ y $\mathbf{W}$ es el peso. Son necesarios por lo menos dos estados límites: para sismos menores, un estado límite de funcionalidad (ELF) que asegure comportamiento quasi-elástico y para los fuertes, un estado límite de seguridad (ELU), con suficiente rigidez y resistencia para limitar el comportamiento inelástico, como prevención de colapso (Lobo Quintero, 1996). Cada estado límite corresponde a niveles de desempeño e introduce grados de confiabilidad expresados por la probabilidad de excedencia y el período de retorno. En las expresiones de la demanda, se incorporan factores basados en experiencia y juicio ingenieril, que introducen un grado de incertidumbre (Collins et al, 1996). Como ejemplo, se aprecia la influencia del factor de importancia $\alpha$ en la probabilidad de excedencia de la aceleración y en los períodos de retorno Tr de Ao (Máxima aceleración esperada a nivel de roca en el sitio, como fracción de g) mostrados en la Tabla 1. El mejor método para establecer la adecuación de un código, es medir el grado de desempeño mediante la probabilidad de alcanzar un estado límite en un período de retorno pre-determinado.

Tabla 1. Influencia del Factor de Importancia en los Períodos de Retorno (Grases, 1987).

\begin{tabular}{|c|c|c|c|c|c|c|c|c|}
\hline \multirow[t]{2}{*}{ LOCALIDAD } & \multirow[t]{2}{*}{ ZONA } & \multirow{2}{*}{ Ao } & \multirow[t]{2}{*}{$\begin{array}{c}\text { FACTOR } \\
\alpha\end{array}$} & \multirow[t]{2}{*}{$\begin{array}{c}\text { VALOR } \\
\alpha . \text { Ao }\end{array}$} & \multicolumn{3}{|c|}{$\begin{array}{l}\text { PROBABILIDAD DE EXCEDEN-CIA de } \\
\text { Ao en T años }\end{array}$} & \multirow[t]{2}{*}{$\begin{array}{c}\text { PERIODO } \\
\text { Tr }\end{array}$} \\
\hline & & & & & $1 \mathrm{AÑO}$ & $50 \mathrm{AÑOS}$ & 100 AÑOS & \\
\hline \multirow{3}{*}{$\begin{array}{c}\text { PELIGRO } \\
\text { MODERADO }\end{array}$} & \multirow{3}{*}{2} & \multirow{3}{*}{0.15} & 1.0 & 0.15 & 0.0021 & 0.101 & 0.192 & 469 \\
\hline & & & 1.2 & 0.18 & 0.0010 & 0.050 & 0.098 & 973 \\
\hline & & & 1.5 & 0.225 & 0.0004 & 0.021 & 0.041 & 2374 \\
\hline \multirow{3}{*}{$\begin{array}{l}\text { PELIGRO } \\
\text { ELEVADO }\end{array}$} & \multirow{3}{*}{4} & \multirow{3}{*}{0.30} & 1.0 & 0.30 & 0.0021 & 0.100 & 0.190 & 473 \\
\hline & & & 1.2 & 0.36 & 0.0012 & 0.059 & 0.115 & 818 \\
\hline & & & 1.5 & 0.45 & 0.0006 & 0.031 & 0.061 & 1597 \\
\hline
\end{tabular}

La concepción probabilística se puede incorporar a través de espectros de riesgo uniforme, donde cada ordenada tiene la misma probabilidad de excedencia y la forma espectral no representa un sismo único. (Collins, 1995). Hay metodologías alternas que trabajan con la curva de frecuencia anual de la aceleración máxima y los espectros medios más una desviación estándar 
$\sigma$ obtenida con un enfoque determinístico en los estudios de sitio (Loh et al, 1994). Estos espectros aún en proceso de desarrollo, son un paso para incorporar las incertidumbres de todas las variables y alcanzar la confiabilidad global del diseño. Los espectros estadísticos suavizan efectos importantes como la influencia de las ondas de Rayleigh para sismos fuertes de subducción (Saragoni et al, 1998). Para relacionar los espectros de aceleración elástica e inelástica, se define el factor de respuesta $\mathbf{R}$ que en la zona de períodos cortos $\mathbf{T}<\mathbf{T o}$ (Período final de la rama ascendente del espectro), depende de variables aleatorias que requieren mayor investigación y que en el rango flexible se toman como $\mathbf{R}=\mu$ :

$$
\mathbf{R}=\mathbf{S a}(\mu=1) / \mathbf{S a}(\mu)=\mathbf{R}\left(\mathbf{p}, \mathbf{T}, \mathbf{T}_{\mathbf{g}}, \mu, \xi, \beta, \mathbf{r}, \Omega, \mathbf{i}, \mathbf{h}, . . .\right) \quad \mathbf{T}<\mathbf{T o}
$$

Donde $\mathbf{p}$ es la probabilidad de excedencia, $\mathbf{T}$ el período estructural, $\mathbf{T}_{\mathbf{g}}$ el período predominante del suelo, $\mu$ la ductilidad, $\xi$ el factor de amortiguamiento, $\beta$ el factor de amplificación del suelo, $\mathbf{r}$ la redundancia, $\Omega$ la sobrerresistencia, i la irregularidad y $\mathbf{h}$ el modelo histerético. $\mathbf{R}$ es la variable más discutida e investigada de la Ingeniería Sísmica, donde no puede justificarse un solo valor, sin incorporar variables geométricas de la estructura; donde probablemente, los valores asignados no producen el desempeño deseable; ni se hacen depender del período, del tipo de suelo y de las reservas de resistencia, tampoco dan un nivel de riesgo uniforme a todos los sistemas estructurales (Bertero, 1986; ATC-19, 1995). La expresión más utilizada de R (ATC32, 1996) es :

$$
\mathbf{R}=\mathbf{1}+\left(\mathbf{T} / \mathbf{T}_{\mathrm{g}}\right)(\boldsymbol{\mu}-\mathbf{1})
$$

Tabla 2. Valores de $T_{g}$ según M (Magnitud Richter), Ao y el tipo de suelo S (ATC-32,1996)

\begin{tabular}{|c|c|c|c|c|c|c|c|c|c|c|c|c|}
\hline \multirow{2}{*}{$\begin{array}{c}\text { go } \\
\mathrm{g}\end{array}$} & \multicolumn{4}{|c|}{$\mathbf{M}=6.5 \pm 0.25$} & \multicolumn{4}{|c|}{$\mathbf{M}=7.25 \pm 0.25$} & \multicolumn{4}{|c|}{$\mathbf{M}=8.0 \pm 0.25$} \\
\cline { 2 - 15 } & $\mathrm{S} 1$ & $\mathrm{~S} 2$ & $\mathrm{~S} 3$ & $\mathrm{~S} 4$ & $\mathrm{~S} 1$ & $\mathrm{~S} 2$ & $\mathrm{~S} 3$ & $\mathrm{~S} 4$ & $\mathrm{~S} 1$ & $\mathrm{~S} 2$ & $\mathrm{~S} 3$ & $\mathrm{~S} 4$ \\
\hline 0.1 & 0.32 & 0.45 & 0.46 & 0.44 & 0.41 & 0.53 & 0.56 & 0.56 & 0.51 & 0.69 & 0.71 & 0.71 \\
\hline 0.2 & 0.37 & 0.44 & 0.49 & 0.64 & 0.42 & 0.53 & 0.55 & 0.74 & 0.47 & 0.61 & 0.65 & 0.85 \\
\hline 0.3 & 0.35 & 0.43 & 0.50 & 0.73 & 0.38 & 0.51 & 0.55 & 0.76 & 0.48 & 0.64 & 0.65 & 0.98 \\
\hline 0.4 & 0.39 & 0.47 & 0.50 & 0.87 & 0.42 & 0.56 & 0.59 & 0.93 & 0.46 & 0.62 & 0.66 & 1.04 \\
\hline 0.5 & 0.37 & 0.46 & 0.50 & -- & 0.42 & 0.53 & 0.62 & -- & 0.45 & 0.59 & 0.70 & -- \\
\hline 0.6 & 0.35 & 0.44 & 0.50 & -- & 0.43 & 0.54 & 0.64 & -- & 0.46 & 0.60 & 0.76 & -- \\
\hline 0.7 & -- & -- & -- & -- & 0.50 & 0.66 & 0.76 & -- & 0.54 & 0.71 & 0.80 & -- \\
\hline
\end{tabular}

Donde S1 corresponde a un suelo firme y S4 al más blando. Algunos limitan el período característico a $\mathbf{0 . 4}$ seg., pero la Tabla 2, da una variedad de períodos predominantes $\mathbf{T}_{\mathbf{g}}$ mayores. Los factores $\mathbf{R}$ deducidos de registros en roca o aluvión, hacen inseguras a las estructuras rígidas sobre suelos blandos, dando valores pequeños de $\mathbf{R}$, pero se incrementan las demandas de ductilidad cuando se aplican valores mayores (Miranda, 1993a). Con excepción de los puentes con aislamiento, de gran luz o colgantes, la gran mayoría tiene períodos menores de 0.6 seg. (Miranda, 1993b). En los métodos de diseño por resistencia MDR, fundamentados en la capacidad de absorción y disipación de energía, el factor $\mathbf{R}$ reduce las fuerzas elásticas hasta un nivel de cedencia significativa y el factor de amplificación de los desplazamientos $\mathbf{C}_{\mathbf{d}}$ reconvierte las deformaciones en no lineales (ATC - 3, 1978), sobre la base de la relación entre el desplazamiento espectral elástico $\mathrm{S}_{\mathrm{de}}$ y el inelástico $\mathrm{S}_{\mathrm{di}}$ 
$S_{\mathrm{di}}=\mathbf{S}_{\mathrm{de}}\left(\mathbf{C}_{\mathrm{d}} / \mathbf{R}\right)$

donde el factor de amplificación del desplazamiento $\mathbf{C}_{\mathbf{d}}$ depende del sistema estructural, de la ductilidad $\mu$, de la sobrerresistencia y de $\mathbf{T}_{\mathbf{g}}$. En general, este método depende mucho de $\mathbf{R}$ y establece secciones de disipación de energía en los extremos de las columnas, donde las relaciones capacidad/demanda, no concuerdan con las demandas de ductilidad y dan una respuesta inelástica no uniforme, que justifique la vigencia del método de diseño elástico. Como bajo acciones sísmicas los desplazamientos son más representativos del comportamiento que las fuerzas, y los daños están ligados íntimamente a las deformaciones, los valores límites impuestos por los códigos permiten controlar el desempeño estructural, quedando la revisión de la resistencia como un paso final. El desplazamiento límite $\delta_{\mathbf{t}}$ para un período efectivo $\mathbf{T}_{\mathbf{e}}$ puede estimarse mediante la siguiente expresión (FEMA, 1996):

$\delta_{t}=C_{0} C_{1} C_{2} C_{3} S_{a}\left(T_{e} / 2 \pi\right)^{2}$

Donde ya se han definido las constantes y $1 \leq \mathbf{C}_{\mathbf{0}} \leq 1.5$ incluye la participación modal. Con estos fundamentos se desarrolla el método de diseño por desplazamientos MDD, conforme a los criterios de desempeño (Qi y Moehle, 1991; Kowalsky et al, 1994; Calvi y Kingsley, 1995; Collins et al, 1996). Para facilitar la aplicación de este método, conviene tener las demandas en aceleración espectral $\mathbf{S}_{\mathrm{a}}$ y en desplazamiento espectral $\mathbf{S}_{\mathrm{d}}$, y más aún en el plano $\mathbf{S}_{\mathrm{a}}-\mathbf{S}_{\mathrm{d}}$, con períodos radiales, tal como se muestra en las Fig. 4(a) y 4(b), adaptadas del ATC-32. En este manual se da una metodología para el MDD en puentes, que incluye el cálculo de $\mathbf{R}$ para una fuerza cortante de fluencia $\mathbf{V}_{\mathbf{y}}$ obtenida de la curva de capacidad y puede determinarse según la expresión siguiente (Ascheim et al, 1997; FEMA, 1996):

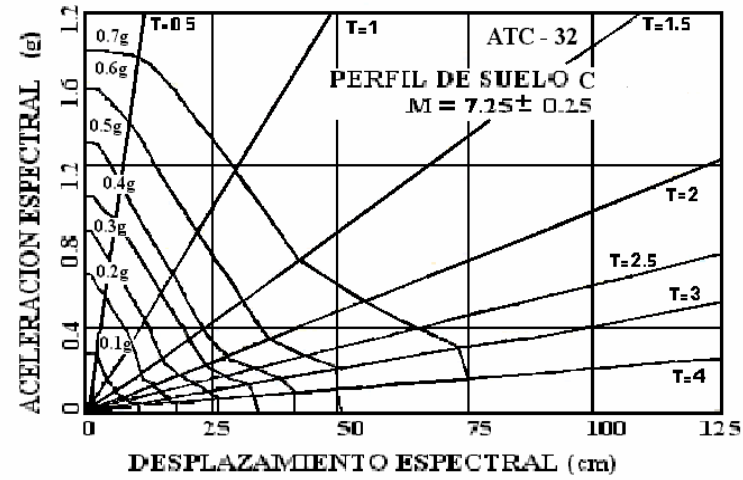

(a)

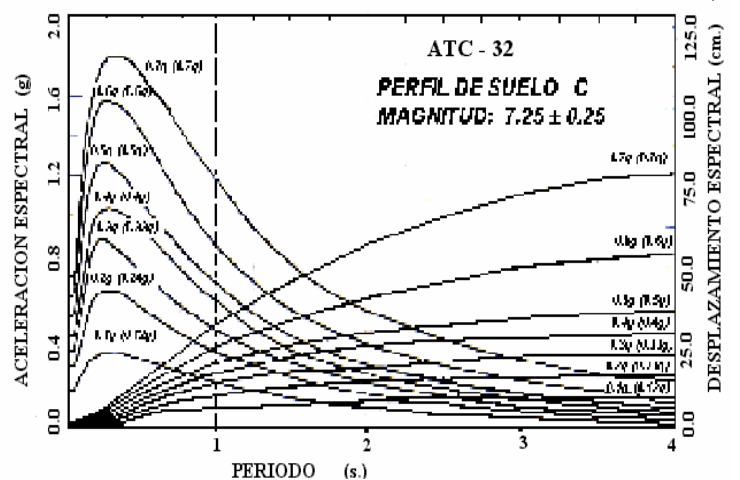

(b)

Fig. 4. Espectros de Aceleración y de Desplazamiento. Espectros Aceleración-Desplazamiento.

$\mathbf{R}=\mathbf{S}_{\mathbf{a}} /\left[\mathbf{C}_{\mathbf{0}}\left(\mathbf{V}_{\mathbf{y}} / \mathbf{W}\right)\right]$

Para el desarrollo de la curva de capacidad representativa del Método Estático No Lineal, el modelo matemático de la estructura se somete a una carga lateral monotónica creciente hasta que sea excedido un desplazamiento límite. Como el modelo toma la respuesta inelástica, las fuerzas internas resultantes deben dar aproximaciones razonables a los efectos de un sismo. En este contexto, deben aplicarse y mantenerse durante el análisis las cargas gravitacionales, las cargas 
laterales Fi siguen una distribución basada en las formas modales, y para el caso de puentes especiales se deben incorporar los modos superiores. Una forma de hacerlo es mediante la siguiente expresión, válida para una masa del modo $\mathbf{k}, \mathbf{m}_{\mathbf{k}}$, una forma $\phi_{\mathbf{k j}} \mathrm{y}$ una aceleración espectral S $\mathbf{S}_{\text {aj }}$ (Ramírez, 1999):

$$
F_{i}=\sqrt{\sum_{j=1}^{N}\left(\left[\frac{\sum_{k=1}^{N} m_{k} \phi_{k j}}{\sum_{k=1}^{N} m_{k} \phi_{k j}^{2}}\right] \phi_{i j} S_{a j} m_{i}\right)^{2}}
$$

Es muy importante en puentes, seleccionar el punto característico $\mathbf{k}$ donde se registran los desplazamientos (análogo al tope de los edificios), el cual se toma sobre el tablero, donde se presenten los valores máximos, quedando el corte basal como la fuerza característica necesaria para determinar la curva de capacidad. Una metodología alterna para puentes, basada en desempeño estructural y desarrollada por Ramírez en 1999, se detalla a continuación: $1^{\circ}$. Determinar los modos más influyentes y la carga lateral equivalente. $2^{\circ}$. Hacer el análisis elástico no lineal, con la distribución de cargas obtenida hasta alcanzar los estados límites preestablecidos. $3^{\circ}$. Con los resultados de este análisis, construir la curva de capacidad $\left(\mathrm{V}-\Delta_{\mathrm{k}}\right)$, modificando gradualmente la forma de carga; Aproximar la curva con una forma bi-lineal en el plano $\left(\mathrm{S}_{\mathrm{a}}-\mathrm{S}_{\mathrm{d}}\right)$, donde:

$$
\begin{array}{cc}
\mathbf{S}_{\mathbf{a}}=\mathbf{V} /\left(\alpha_{\mathbf{j}} \mathbf{W}\right) & \mathbf{y} \quad \mathbf{S}_{\mathbf{d}}=\Delta_{\mathbf{k}} /\left(\Gamma_{\mathbf{i j}}\right), \\
\left.\Gamma_{i j}=\frac{\left[\sum_{k=1}^{N} m_{k} \phi_{k j}\right.}{\sum_{k}^{N} m_{k} \phi_{k j}^{2}}\right]_{i j} & \alpha_{j}=\frac{\left[\sum_{k=1}^{N} m_{k} \phi_{k j}\right]^{2}}{\left[\sum_{k=1}^{N} m_{k}\right] \sum_{k=1}^{N} m_{k} \phi_{k j}^{2}}
\end{array}
$$

$4^{\text {o. }}$ Determinar la masa modal equivalente a un sistema de un grado, según $\mathbf{m}_{\text {eq• }}=\Sigma \mathbf{m}_{\mathbf{i}} \phi_{\mathbf{i}}{ }^{2}$

$5^{\circ}$. Obtener la curva de comportamiento $\left(\mathbf{V}_{\mathbf{m}}-\mathbf{S}_{\mathbf{d}}\right)$ equivalente a un grado de libertad, siendo $\mathbf{V}_{\mathbf{m}}=\mathbf{S}_{\mathbf{a}} \cdot \mathbf{m}_{\mathrm{eq}}$.

$6^{\circ}$. Si se toman modos superiores, la curva de comportamiento se basa en el modo dominante, transformando sus coordenadas a través de $\alpha \mathbf{1}$ (ecuación 8), así:

$\Delta \mathbf{1}=\alpha \mathbf{1} \Delta_{\mathrm{n}} ; \mathrm{y} \mathbf{V 1}=\alpha \mathbf{1} \mathbf{V n}$ (desplazamiento $\Delta$ y corte $\mathbf{V}$ en el modo 1 y en el modo n).

$7^{\circ}$. Se realiza un análisis dinámico no lineal del sistema equivalente de un grado, para determinar el máximo valor espectral $\mathbf{S}_{\mathbf{d}} \quad$ que corresponde al punto característico, usando como demanda uno o varios registros sísmicos.

$8^{\circ}$. Se transfoma este valor en $\Delta_{\mathbf{k}}=\mathbf{S}_{\mathbf{d}} \cdot \Gamma_{\mathbf{k 1}}$. Tomando en cuenta el cálculo de distribución de cargas seleccionado ( $\mathrm{k} 1$ es el punto de comportamiento).

$9^{\circ}$. Se hace una nueva curva de capacidad hasta un desplazamiento máximo $\Delta_{\mathbf{k}}$ obtenido en el paso 7 . 
El MDD facilita el diseño para dos estados límites ELF y ELU, y para métodos basados en desempeño que son los más modernos y avanzados (SEAOC Visión 2000, 1995; Krawinkler, 1999), se adapta a la solución de sistemas de varios grados de libertad, mediante métodos de estructuras equivalentes a un grado de libertad (Gulkan y Sozen, 1974; Shibata y Sozen, 1976; Qi y Moehle, 1991); y se pueden interceptar los espectros inelásticos o los construidos con amortiguamientos distintos con las curvas de capacidad, sobre lo cual se han tenido experiencias favorables en edificaciones (Freeman, 1978; Pincheira, 1993), pero que deben adaptarse a la condición particular de los puentes (Ramírez, 1999).

\section{ANÁLISIS ELÁSTICO LINEAL}

Partiendo de la ecuación de equilibrio dinámico para un sistema de $\mathbf{n}$ grados de libertad:

\section{$[\mathbf{M}]\{\mathbf{i}\}+[C]\{\dot{\mathbf{u}}\}+[\mathbf{K}]\{\mathbf{u}\}=-[\mathbf{M}][\mathbf{I}]\{\ddot{\mathbf{u}} \mathbf{g}\}$}

donde, se tienen las matrices de masas $[\mathbf{M}]$, de amortiguamiento $[\mathbf{C}]$ y de rigideces $[\mathbf{K}]$ asociadas a los grados de libertad $\{\mathbf{u}\}$; siendo $\left\{\ddot{\mathbf{u}}_{\mathbf{g}}\right\}$, la aceleración de la base. El método de análisis dinámico multimodal ADM que considera seis grados de libertad nodal, se asume para puentes de configuración irregular y está al alcance de la práctica profesional. Además, se aplica en puentes esviados, curvos, con tramos desbalanceados o con articulaciones intermedias.

El comportamiento de los puentes regulares se asimila a sistemas que responden en primer modo. La deflexión dinámica $\mathbf{u}(\mathbf{x , t})$ se aproxima mediante una función de desplazamientos:

$\mathbf{u}(\mathbf{x}, \mathbf{t})=\mathbf{u}_{\mathbf{s}}(\mathbf{x}) \cdot \mathbf{u}(\mathbf{t})$

donde $\mathbf{u}_{\mathbf{s}}(\mathbf{x})$ es la función aproximada del primer modo obtenida bajo acciones estáticas supuestas $\mathbf{p}_{\mathbf{o}} ; \mathbf{u}(\mathbf{t})$ es la variable correspondiente al sistema de un grado de libertad. El término de carga depende del peso distribuido del tablero $\mathbf{w}(\mathbf{x})$, de la deformación $\mathbf{u}_{\mathbf{s}}(\mathbf{x})$ y de la aceleración constante $\ddot{\mathbf{u}}_{\mathrm{g}}(\mathrm{t})$ :

$$
\ddot{u}(t)+2 \xi \dot{u}(t)+\omega^{2} u(t)=(\beta / \gamma) \ddot{u}_{g}(t)
$$

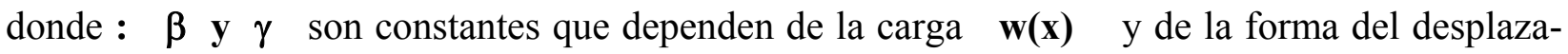
miento asumido $\mathbf{u}_{\mathbf{s}}(\mathbf{x})$. La carga que corresponde al Análisis Estático Equivalente AEE resulta ser:

$\mathbf{p}_{\mathbf{e}}(\mathbf{x})=(\beta / \gamma) \cdot \mathbf{A}_{\mathbf{d}} \cdot(\xi, T) \cdot \mathbf{g} \cdot \mathbf{w}(\mathbf{x}) \cdot \mathbf{u}_{\mathbf{s}}(\mathbf{x})$

Donde $\mathbf{A}_{\mathbf{d}}(\boldsymbol{\xi}, \mathbf{T})$ es la aceleración espectral de diseño para una razón de amortiguamiento $\xi$. Este método es válido para puentes rectos de uno o dos tramos, que responden como puentes rígidos, tienen esviaje muy pequeño y su rigidez es controlada por los estribos (ATC 32, 1996).

Un intento para cubrir los efectos de las excitaciones espectrales múltiples en la base de los apoyos; por ejemplo en tres apoyos 1,2,3, con movimientos en la base $\mathbf{u}_{\mathbf{g 1}}, \mathbf{u}_{\mathbf{g 2}}, \mathbf{u}_{\mathbf{g} 3}$ para sistemas de un grado de libertad; con diafragma rígido y rigideces de las pilas $\mathbf{K}_{\mathbf{1}} \mathbf{K}_{\mathbf{2}} \mathbf{K}_{\mathbf{3}}$, se adapta de Kawashima, 1994:

$$
m \cdot \ddot{u}_{t}(t)+C \dot{u}_{t}(t)+(S K i) \cdot u_{t}(t)-\left(K 1 u_{g 1}+K 2 u_{g 2}+K 3 u_{g 3}\right)=0
$$


donde: $\quad \mathbf{u}_{\mathbf{t}}(\mathbf{t})=\mathbf{u}_{\mathbf{s}}+\mathbf{u}$ (deformación estática + deformación dinámica);

$$
u_{s}=(1 / \Sigma K i) \cdot\left(K_{1} u_{g 1}+K_{2} u_{g 2}+K_{3} u_{g 3}\right)
$$

se obtienen los espectros de respuesta múltiples que son efectivos para estructuras rígidas de período corto, aunque las respuestas de aceleración son menores. Se asegura que las respuestas bajo efectos no-sincrónicos pueden ser mayores o menores que las uniformes y esto depende de las características estructurales, sísmicas y geotécnicas. El parámetro más importante es la frecuencia dominante del sitio, pero si existen diferencias significativas entre dos apoyos, esta incoherencia obliga a tener una regla más precisa de superposición modal. Der Kiureghian et al 1997, definen el concepto de Variabilidad Espacial como producto de cuatro efectos: incoherencia por dispersión de ondas en medios heterogéneos; paso de ondas por la diferencia en el tiempo de llegada en varias estaciones; atenuación o decaimiento de amplitudes con la distancia por acción geométrica o disipación de energía y efecto de sitio, por la influencia del perfil de suelos en el contenido frecuencial y la amplitud en cada estación. Se demuestra que el método de la envolvente espectral puede producir grandes errores y resultados inseguros.

Aplicando la ecuación (9) mediante la teoría de vibraciones aleatorias no estacionarias Der Kiureghian et al 1997, presentan una regla y un programa de combinación de respuestas múltiples en el campo elástico, que consta de tres términos: el primero, las componentes pseudo estáticas representadas por una doble suma sobre los grados de libertad de los apoyos; el

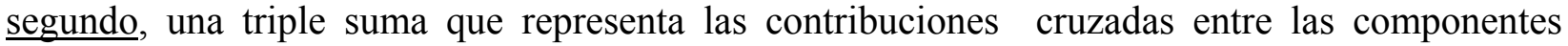
estáticas y dinámicas y el último una suma cuádruple para representar las componentes dinámicas de la respuestas de los apoyos libres. La expresión es la siguiente:

$$
\begin{aligned}
& \mathrm{E}[\operatorname{máx}|z(t)|]=\left[\sum_{k=1}^{m} \sum_{i=1}^{m} a_{k} a_{i} \rho_{u_{k} u_{i}} u_{k, \text { máx }} u_{i, \text { máx }}\right. \\
& +2 \sum_{x=1}^{m} \sum_{i=1}^{m} \sum_{j=1}^{n} a_{k} b_{\mathrm{lj}} \rho_{u_{k} s_{j}} u_{k, \text { máx }} D_{i}\left(\omega_{j}, \zeta_{j}\right) \\
& \left.+\sum_{k=1}^{m} \sum_{i=1}^{m} \sum_{i=1}^{n} \sum_{j=1}^{n} b_{k i} b_{i j} \rho_{s_{R i} j_{j}} D_{k}\left(\omega_{i}, \xi_{j i}\right) D_{i}\left(\omega_{j}, \xi_{j j}\right)\right]^{1 / 2}
\end{aligned}
$$

Donde, $\boldsymbol{E}[\mathbf{m a ́ x}|\boldsymbol{z}(\boldsymbol{t})|]$ es la esperanza de la respuesta máxima en valor absoluto, $\mathbf{m}$ es el número de grados de libertad de los apoyos, $\mathbf{n}$ el número de modos, $\mathbf{a}_{\mathbf{k}}$ es el coeficiente de influencia asociado al grado de libertad $\mathbf{k}, \mathbf{b}_{\mathbf{k i}}$ es el factor de participación modal en el apoyo $\mathbf{k}$ y modo $\mathbf{i}$, $\mathbf{u}_{\mathbf{k}, \mathbf{m a ́ x}}$ es el desplazamiento medio del terreno en el apoyo $\mathbf{k}, \mathbf{D}_{\mathbf{k}}\left(\omega_{\mathbf{i}}, \boldsymbol{\xi}_{\mathbf{i}}\right)$ la ordenada del desplazamiento espectral asociada al apoyo $\mathbf{k}$, y a la frecuencia $\omega_{\mathbf{i}}$, y $\xi_{\mathbf{i}}$ es el amortiguamiento del modo i, $\rho_{u_{k} u_{l}}$ el coeficiente de correlación cruzada entre los apoyos $\mathbf{k}$ y $\mathbf{l}, \rho_{u_{k} s_{l_{j}}}$ el coeficiente de correlación cruzada entre el apoyo $\mathbf{k}$, las respuestas del modo $\mathbf{j}$ sujetas al movimiento del apoyo $\mathbf{l}$, y $\rho_{s_{k i} s_{j j}}$ el coeficiente de correlación cruzada entre las frecuencias y los amortiguamientos en los modos $\mathbf{i}$, j sujetos a los movimientos de los apoyos $\mathbf{k}, \mathbf{l}$. 
Las limitaciones del método están en el comportamiento lineal, el amortiguamiento viscoso y los modos normales de vibración. Además se requiere que tengan incoherencia perfecta, o que cualquier par de componentes del movimiento en direcciones ortogonales sean estadísticamente independientes; no se toma en cuenta la interacción suelo-estructura. Se proponen estos aspectos a investigación y estudio incluyendo el cálculo de los coeficientes $\mathbf{a}_{\mathbf{k}} \mathbf{y} \mathbf{b}_{\mathbf{k}}$ así como el número de modos requerido, donde no se deben aplicar criterios de edificios. Se ha presentado un programa de análisis tridimensional no lineal que incorpora el efecto de la variabilidad espacial, en combinación con métodos basados en desempeño estructural (Ramírez y Ayala, 1999b).

\section{ESTRIBOS}

Estos sirven para transferir las fuerzas de la superestructura, son elementos de retención de tierra, constituyen un diafragma extremo y con sus aleros se logra una transición suave. Deben tener suficiente resistencia y rigidez a las fuerzas laterales, mantener los tramos extremos para garantizar el acceso al puente, evitar el daño estructural e incluso el colapso (ATC 18, 1997).

Los estribos pueden estar separados de la superestructura con juntas o fabricados integralmente con ella. Hoy se considera económico y apropiado evitar las juntas para puentes de luces largas o de formas geométricas complicadas utilizando el concepto del estribo integral, el cual se construye monolítico con la super-estructura y con los pilotes de apoyo. Cuando se permiten los movimientos relativos, es semi-integral (Park, 1996). La participación del estribo en la respuesta dinámica global, refleja el comportamiento de la estructura, el mecanismo de transferencia de carga, la rigidez efectiva, la capacidad del muro y el nivel de daños esperado (ATC 32, 1996). La presencia de estribos con juntas, representa una fuente de respuesta no lineal asociada al comportamiento de apoyos y de conectores, la apertura y cierre de juntas de expansión, la resistencia pasiva del terreno, los dentellones de la base, comportamiento de cabezales y la rigidez de pilotes individuales o de grupos. Los empujes laterales del relleno, se evalúan mediante la fórmula de Mononobe-Okabe, deducida para empujes activos (Okamoto, 1973).

En MTC, 1987 se separan los componentes del empuje lateral en estático y dinámico, en función de los factores de presión lateral correspondiente, según su condición de equilibrio plástico: $\mathbf{K}_{\mathbf{a}}$ (activa), $\mathbf{K}_{\mathbf{i}}$ (activa parcial), $\mathbf{K}_{\mathbf{0}}$ (reposo), $\mathbf{K}_{\mathbf{p}}$ ( pasiva), $\mathbf{K}_{\mathbf{a s}}$ (activa sísmica) $\mathbf{y}$ $\mathbf{K}_{\mathbf{p s}}$ (pasiva sísmica), de la aceleración máxima $\mathbf{A}_{\mathbf{0}}$, del corficiente sísmico vertical $\mathbf{C}_{\mathbf{s v}} \mathrm{y}$ del peso unitario $\gamma$, tal como se muestra en la Tabla 3. Esta materia tiene un buen grado de incertidumbre y requiere de más investigación teórica, experimental e instrumentación de campo.

Tabla 3. Componentes del Empuje Lateral

\begin{tabular}{|c|c|c|c|}
\hline $\begin{array}{c}\text { Condición de } \\
\text { equilibrio }\end{array}$ & $\begin{array}{c}\text { Desplaz. } \\
\text { Máximo }\end{array}$ & $\begin{array}{c}\text { Empuje } \\
\text { Estático }\end{array}$ & Incremento de Presión Dinámica \\
\hline ACTIVO & $>0.005 \mathrm{H}$ & $1 / 2 \gamma \mathrm{H}^{2} \mathrm{~K}_{\mathrm{a}}$ & $\mathrm{E}_{\mathrm{ad}}=1 / 2 \gamma \mathrm{H}^{2}\left(\mathrm{~K}_{\mathrm{as}}-\mathrm{K}_{\mathrm{a}}\right)\left(1-\mathrm{C}_{\mathrm{sv}}\right)$ \\
\hline INTERMEDIO & $>0.001 \mathrm{H}$ & $1 / 2 \gamma \mathrm{H}^{2} \mathrm{~K}_{\mathrm{i}}$ & $\mathrm{E}_{\mathrm{id}}=3 / 4 \mathrm{~A}_{\mathrm{o}} \gamma \mathrm{H}^{2}$ \\
\hline NEUTRO & $<0.001 \mathrm{H}$ & $1 / 2 \gamma \mathrm{H}^{2} \mathrm{~K}_{\mathrm{o}}$ & $\mathrm{E}_{\mathrm{od}}=\mathrm{A}_{\mathrm{o}} \gamma \mathrm{H}^{2}$ \\
\hline PASIVO & $>0.01 \mathrm{H}$ & $1 / 2 \gamma \mathrm{H}^{2} \mathrm{~K}_{\mathrm{p}}$ & $\mathrm{E}_{\mathrm{pd}}=1 / 2 \gamma \mathrm{H}^{2}\left(\mathrm{~K}_{\mathrm{ps}}-\mathrm{K}_{\mathrm{p}}\right)\left(1-\mathrm{C}_{\mathrm{sv}}\right)$ \\
\hline
\end{tabular}


Las fuerzas de diseño se ajustan a cada tipo y condición de estribo y para todos los casos deben incorporarse las fuerzas de inercia. La rigidez del estribo está caracterizada por la conexión estructura-estribo, la parte de relleno movilizada, la altura efectiva del estribo, la capacidad de soporte del terreno en estribos y aletas, la capacidad cortante del relleno, el desplazamiento para movilizar empuje pasivo, y para cerrar las juntas, el ángulo de esviaje. Los puentes esviados aumentan su desplazabilidad debido al acoplamiento entre las respuestas longitudinal y transversal, abriendo la junta de expansión. Los modelos de análisis deben tomar en cuenta la resistencia y la rigidez que causan la apertura y el cierre de las juntas; así se tienen modelos de tracción y de compresión.

Existe un posible mecanismo de cedencia en las cabezas de los pilotes, que involucra el desarrollo de una cuña en pasivo de la masa del suelo, que en la dirección transversal depende de los aleros y de los bloques de cortante. La rigidez del estribo se toma como resortes en paralelo que incorporan la rigidez de los pilotes con la desarrollada en el mecanismo de falla (Maroney y Chai, 1994). La determinación de los empujes reales en los estados límites del relleno y su punto de aplicación; la distribución adecuada de la fuerza a los elementos portantes según su deformabilidad; la estabilidad de taludes naturales, banqueos, rocas o terraplenes; el comportamiento sísmico de muros atirantados y de tierra reforzada; el asentamiento de rellenos y losas acceso; y la capacidad portante y de deformabilidad de fundaciones directas, sobre cajones o pilotes, son materias que requieren mayor estudio e investigación.

\section{AISLAMIENTO SÍSMICO Y DISIPACION DE ENERGIA}

El diseño de puentes nuevos y la rehabilitación de puentes existentes requiere de las técnicas protectoras sismo-aislantes y sismo-amortiguantes, sin requerir costosos detalles de ductilidad. Aunque los sistemas sismorresistentes se seguirán aplicando por su conocida practicidad tecnológica, van cediendo terreno a los sistemas de aislamiento y de disipación de energía, ya que para el aislamiento sísmico de puentes, existe una experiencia bien ganada con el uso de apoyos de neopreno (Lobo Quintero, 1998). El aislamiento posibilita la construcción de puentes con tableros continuos de múltiples tramos, eliminando juntas que crean vibración, polución de ruido, hacen al puente no confortable y necesitan cambiarse con frecuencia. Se usan dispositivos que deben ser probados con pruebas dinámicas que confirmen la rigidez equivalente, la estabilidad a cargas reversibles, la existencia de desplazamientos residuales y los cambios por carga cíclica, temperatura y ambiente (Kawashima et al, 1994). Las pruebas pueden hacerse sobre aisladores individuales, pero existen sistemas híbridos pseudo-dinámicos que permiten investigar el comportamiento lineal y no lineal de las pilas. Además, los aisladores quedan sometidos a carga axial y a rotación variable. Después del sismo de Kobe, estos aspectos son motivo de mayor investigación (Igarashi y Iemura, 1996).

Un estudio comparativo de la respuesta de puentes convencionales, puentes con disipación y puentes con aislamiento, reveló que los primeros experimentan altos daños bajo excitaciones sísmicas fuertes y aún bajo efectos ligeros no sincrónicos (Nuti y Ochat, 1994). Además, la adopción de disipadores como fusibles en el tope de las pilas resulta una buena solución. Pero, como el control pasivo está en evolución, se requiere clarificar la definición del estado límite de funcionalidad y los criterios para la evaluación de los desplazamientos. El uso de aisladores de goma es menos frecuente, con la ventaja de aplicarse en puentes irregulares para 
evitar concentraciones de cortante, absorber las deformaciones por temperatura y flujo plástico, y reducir los problemas de direccionalidad. Aun cuando pueden generarse programas de investigación tecnológica para la creación de nuevos elementos de aislamiento y disipación, en nuestra condición de usuarios de estos sistemas, bien merece la pena realizar estudios teóricos y experimentales para conocer mejor su comportamiento, incorporar la amenaza sísmica regional y los estudios de sitio, tener programas de procesamiento adecuado y establecer las limitaciones normativas correspondientes a nuestra construcción, experiencia y realidad. (Tena Colunga, 1997)

\section{NORMATIVA SISMICA}

Un trabajo reciente (Lobo Quintero, 1999) que busque la revisión y modernización de la propuesta de Venezuela MTC-87, se hizo sobre las siguientes bases: 1). Ajustarse en cuanto sea posible a la filosofía de diseño, establecida por las Normas aplicables obligatoriamente al país (AASHTO, 1995). 2). Seguir un formato similar a las Normas Covenin 1756-98 para Edificaciones Sismorresistentes, con el objeto de facilitar su aplicación, y establecer su homología y diferenciación. 3). Adaptar los conceptos recogidos de la experiencia sísmica, de la investigación, de la experimentación, y de la práctica, en beneficio del desarrollo científico y tecnológico de una nueva norma. 4). Procurar la integridad, la continuidad y la redundancia estructural, la limitación del desplazamiento y el detallado del diseño, mediante normas conceptuales, prácticas y modernas, que impulsen su aprobación, legalización y aplicación.

La actualización de estas Normas, requiere de la consideración de los siguientes aspectos:

1. La definición de una filosofía de normas con dos estados límites, que controle el diseño sísmico y determine zonificación, acciones sísmicas, clasificación de sistemas estructurales, métodos de análisis, diseño, evaluación y detallado.

2. La modificación del mapa de zonificación sísmica, para adaptarlo a la sismicidad actualizada, a la probabilidad esperada y a la filosofía de diseño mencionada.

3. La revisión de las formas espectrales y de la clasificación de los suelos, según las investigaciones y las experiencias de comportamiento sísmico.

4. La reafirmación de los MDD, buscando un enfoque híbrido, que permita simultáneamente un control de las intensidades de las fuerzas sísmicas y de los daños esperados.

5. La normalización del sismo-aislamiento y del sismo-amortiguamiento, como medios para evitar la pérdida de vidas, controlar y reducir los daños y mantener la función específica de los puentes.

El ATC - 18, 1997 propone la inclusión de criterios de desempeño sobre la integración de técnicas y de costos discutidos y aprobados por grupos científicos, legislativos y no-técnicos. Para ello, se requiere resolver los siguientes aspectos: la excedencia de los valores determinísticos sobre los probabilísticos en algunas zonas; la incorporación de una categoría "crítica" de puentes que tenga requerimientos financieros; asegurar que el estado límite de funcionalidad permita un servicio inmediato de los puentes después de un sismo sin necesidad de apuntalamiento; definir sismos de referencia para los niveles de funcionalidad y de seguridad; $y$ desarrollar el concepto de "daño significativo" para detectar el número de réplicas que puede resistir la estructura. Además, deben incorporarse en el peligro sísmico los efectos de sismos de 
fuentes cercanas, ya que los efectos pulsativos tienen considerable influencia en la cedencia estructural.

\section{INVESTIGACIÓN FUTURA}

Según la experiencia japonesa, las materias que requieren mayor estudio e investigación, son: la determinación de acciones sísmicas provenientes de fuentes cercanas; definición de daños tolerables y de la importancia, según los costos de construcción y reparación y la probabilidad de los daños; mayor experimentación en ductilidad de pilas; programas de análisis dinámico no lineal incorporando fuentes superficiales; determinación de más soluciones de aislamiento sísmico de puentes nuevos y existentes; determinación de la capacidad y deformabilidad de los apoyos elastoméricos; desarrollo de dispositivos preventivos para la caída de tramos; y la incorporación de los efectos de licuación y desprendimiento lateral en suelos blandos saturados y rellenos (Iwasaki, 1996).

El taller de trabajo acerca de las metodologías de diseño sísmico necesarias para la generación de los próximos códigos, realizado en Bled, Slovenia en junio de 1997, determinó el futuro de la ingeniería sísmica, basada en criterios de desempeño, para lo cual se requiere el desarrollo de procedimientos con base probabilística que involucren las demandas sísmicas, los criterios de resistencia, capacidad y deformación a diferentes niveles, para elementos estructurales y no estructurales, y sistemas completos de estructura-suelo-fundación (Fajfar y Krawinkler, 1997). La ingeniería basada en desempeño, se define como un esfuerzo integrado de diseño, construcción y mantenimiento para producir edificaciones con objetivos de desempeño predecible. Se recomienda una implementación gradual del diseño por desplazamientos controlados, con tres etapas: $1^{\mathrm{o}}$. Diseño por fuerzas confrontado con la verificación de deformaciones; $2^{\circ}$. Coexistencia de ambos métodos, con predominio del segundo, y $3^{\circ}$. Adopción directa del MDD (Bertero, 1997). En el área de puentes, se considera correctos los avances experimentados por las normas actuales, aunque subsisten casos en donde los procedimientos fallan, y se requieren nuevas metodologías de diseño. Se identifican algunas carencias de la práctica: a). Como MDR es el método más aplicado, pueden identificarse muchos puentes que no justifican el factor de respuesta $\mathrm{R}$ asignado; b). Se necesita mejorar la definición y los niveles de la acción sísmica; c). Como algunos códigos son muy prescriptivos, se requiere liberar un poco más a los diseñadores; d). No hay consenso en los procedimientos analíticos. e). Predomina el enfoque en ELU y un solo sismo de diseño. e). Los códigos de puentes se han derivado de la experiencia de edificios.

Para identificar el comportamiento de los puentes, es suficiente con tres niveles de desempeño: operacional sin interrupción del tráfico, operacional con daños menores y nivel cercano al colapso. La intensidad sísmica y la importancia del puente deben incorporarse en los objetivos. Existe un acuerdo general en el uso de MDD, pero no hay una definición metodológica; aunque por su simplicidad, comprensión física y mayor control de los resultados, se prefieren los métodos elásticos equivalentes. Los criterios para un diseño conceptual basado en desempeño están por definirse; la influencia del sismo debe incorporarse inicialmente, ya que la práctica lo considera después del diseño por carga vertical. 
Los tópicos de investigación son los siguientes: mejorar la descripción y cuantificación de la demanda sísmica, en particular para estructuras de gran luz; definir más racionalmente los factores de respuesta R; establecer la aplicabilidad y necesidad de diferentes técnicas de análisis con varios niveles de sofisticación; definir los criterios de desempeño con la evidencia de sismos ocurridos y estudios analíticos y experimentales; identificar los criterios de desempeño en áreas de moderada sismicidad; investigar el impacto socio-económico de incluir la respuesta sísmica para la vida de diseño del puente; desarrollar procedimientos simples para tomar en cuenta la interacción suelo estructura de pilas y estribos; realizar investigación analítica y experimental en componentes; e investigar los efectos impuestos de carácter no sincrónicos en la respuesta de puentes largos.

En los Estados Unidos, la investigación se dirige hacia la evaluación de la respuesta inelástica 3-D usando MDD; a la caracterización del enfoque de diseño y las respuestas a sismos de fuentes cercanas; al desarrollo de relaciones de degradación de resistencia al cortante de columnas; al estudio de la influencia de la aceleración vertical en la rigidez y resistencia de columnas; y a la integración de las incertidumbres de los movimientos y respuestas bajo la concepción del desempeño estructural (Aschheim et al, 1997).

\section{CONCLUSIONES Y RECOMENDACIONES}

Para aplicar el estado del conocimiento al contexto regional, se proponen recomendaciones que mejoren su normalización, diseño, experimentación, construcción, control de carga y rehabilitación, donde por su importancia y costo la prioridad presente y futura la tienen los programas de evaluación y reforzamiento de puentes:

a) Los puentes son estructuras de características especiales particularmente vulnerables, que requieren ser diseñados, mantenidos, evaluados y rehabilitados bajo normativas sísmicas actualizadas para una función social y un servicio permanente.

b) Se requiere realizar estudios de amenaza sísmica regional que determinen las aceleraciones probables y los períodos de retorno correspondientes a diferentes condiciones límites de diseño, a fin de fortalecer los métodos basados en el desempeño estructural.

c) Deben determinarse espectros de aceleración, velocidad y desplazamiento bajo la concepción de riesgo uniforme aplicando sismos reales a diferentes perfiles de suelo e incorporando efectos sísmicos producidos por fuentes cercanas.

d) Son necesarios más estudios e investigaciones para determinar factores de respuesta $\mathrm{R}$ que correspondan a la capacidad de absorción y disipación de energía de las estructuras de puentes tomando en cuenta las variables sismológicas, geotécnicas y estructurales.

e) Para controlar mejor el desempeño estructural, utilizando curvas de capacidad, ductilidad esperada y métodos de análisis que se adapten específicamente a las estructuras de puentes.

f) La elaboración de las curvas de capacidad debe ser reglamentada para que se defina el punto característico de la respuesta, la forma de distribución de las cargas, la influencia de los modos superiores y las correcciones para su adaptación a la escala espectral.

g) Es necesario poner a la disposición de los proyectistas de puentes programas completos de análisis lineal y no lineal, que incorporen la respuesta tri-dimensional, la variabilidad espacial, el control del desempeño estructural y la influencia de estribos, apoyos, juntas, aisladores y disipadores de energía. 
h) Para adquirir alguna experiencia real sobre los desarrollos teóricos de la variabilidad espacial, se requiere realizar programas de instrumentación de sistemas completos suelo, fundación y estructura de puentes de múltiples tramos ubicados en zonas de alta sismicidad.

i) Hacen falta estudios experimentales de campo y mediciones in situ de diferentes tipos de estribos, bajo excitación sísmica, para conocer los coeficientes reales del empuje lateral, dentro de los estados de equilibrio límite del relleno y su contribución a la rigidez y resistencia del conjunto estructura-estribo.

j) Se requiere preparar normas regionales de diseño de puentes nuevos y mantenimiento, evaluación y rehabilitación de puentes existentes, adaptadas según la concepción sísmica más reciente, que incorporen la experiencia particular en comportamiento de puentes.

k) Siguiendo el curso de los éxitos y los fracasos de los puentes ante las solicitaciones sísmicas, debe darse mayor prioridad a la concepción de un sistema estructural integral, con deformación controlada, configuración sencilla, preferiblemente hiperestática, detalles constructivos adecuados y fundaciones seguras.

\section{REFERENCIAS}

AASHTO-LRFD (1994), Bridge design specifications, primera edición.

AASHTO (1995) Standard specification for highway bridges, decimosexta edición.

Aschheim M., Moehle, J.R. y Mahin, S. A. (1997), "Design and evaluation of reinforced concrete bridges for seismic resistance", Reporte No. UCB/EERC-97/04, Earthquake Engineering Research Center, University of California at Berkeley.

ATC-3 (1978), "Tentative provisions for the development of seismic regulations for buildings", Applied Technology Council, Redwood City, CA, junio.

ATC-18 (1997), "Seismic criteria for bridges and other highway structures: current and future", Applied Technology Council, Redwood City, CA.

ATC-19 (1995), "Structural response modification factors", Applied Technology Council, Redwood City, CA.

ATC-32 (1996), "Improved seismic design criteria for California bridges: provisional recommendations", Applied Technology Council, Redwood City, CA.

Bertero, V. V. (1986), "Evaluation of response reduction factors recommended by ATC and SEAOC", Memorias, Third U.S. National Conference on Earthquake Engineering, Charleston, N.C.

Bertero, V.V. (1997), "Performance-based seismic engineering: A critical review of proposed guidelines", Seismic design methodologies for the next generation of codes, Bled, Slovenia, A. A .Balkema. Rotterdam. Brookfield.

Buckle, I. G. (1996), "Overview of seismic design methods for bridges in different countries and future directions", Memorias, XI World Conference on Eartquake. Engineering, Acapulco, México, Artículo No. 2113, CDROM. 
Calvi, G. M. y Kingsley, G. R. (1995), "Displacement-based seismic design of multi-degree-offreedom bridge structures", Earthquake. Engineering and Structural.Dynamics, Vol. 24, pp 1247-1266.

Calvi, G. M. y Pinto, P. E. (1996), "Assessment of EC8 provisions for reinforced concrete bridges", Memorias, XI World Conference on Eartquake. Engineering, Acapulco, México, Artículo No. 2052, CDROM.

Camargo, M. R. (1997) “Revisión de la troncal uno, estados Trujillo, Mérida y Táchira”, Informe, comunicación personal.

Collins, K. R. (1995), "A reliability-based dual level seismic design procedure for building structures", Earthquake Spectra, Vol. 11, No. 3, agosto.

Collins K. R., Wen, Y-K y Foutch, D. A. (1996), "Dual-level seismic design: A reliability-based methodology", Earthquake Engineering and Structural Dynamics, Vol. 25, pp. 1433-1467.

Covenin 1756-98 (1998), Norma de Edificaciones Sismorresistentes, Ministerio de Desarrollo Urbano, Fondonorma, Venezuela.

Der Kiureghian, A., Keshishian, P. y Hakobian, A. (1997), "Múltiple support response spectrum analysis of bridges including the site-response effect, the MSRS code", Reporte UCB/EERC97/02, Earthquake Engineering Research Center, University of California at Berkeley.

Fajfar, P. y Krawinkler, H. Editores (1997), Seismic design methodologies for the next generation of codes, A.A.Balkema. Rotterdam. Brookfield.

FEMA-273 (1996), Guidelines for the Seismic Rehabilitation of Buildings, BSSC, septiembre.

Freeman, S. A. (1978), "Prediction of response of concrete buildings to severe earthquake motion", Memorias, Douglas McHenry International Symosium. on Concrete Structures, SP-55, ACI.

Grases, J. (1987), Concreto armado en zonas sísmicas, Divisider, Sivensa, Venezuela.

Gulkan, P. y Sozen, M. A. (1974), "Inelastic responses of reinforced concrete structures to earthquake motions", Journal of American Concrete Institute, 604-610, diciembre.

Igarashi, A. y Iemura, H. (1996), "Experimental and analitycal evaluation of seismic performance of highway bridges with base isolation bearings", Memorias, XI World Conference on Eartquake. Engineering, Acapulco, México, Artículo No. 553, CDROM.

Iwasaki, T. (1996), "Perspectives of seismic design criteria for highway bridges in Japan", Memorias, XI World Conference on Eartquake. Engineering, Acapulco, México, Artículo No. 2110, CDROM.

Kawashima, K. (1994a), "Seismic design force of bridges", Memorias, Second International Workshop on Seismic Design and Retrofitting of Reinforced Concrete Bridges, University of Canterbury, Queenstown, Nueva Zelanda.

Kawashima, K., Unjoh, S. y Sugita, H. (1994b), "Menshin design of highway bridges", Memorias, Second International Workshop on Seismic Design and Retrofitting of Reinforced Concrete Bridges, University of Canterbury, Queenstown, Nueva Zelanda. 
Kowalsky, M. J., Priestley, M. .J. N. y MacRae, G. A. (1994), "Displacement based design of RC bridge columns", Memorias, Second International Workshop on Seismic Design and Retrofitting of Reinforced Concrete Bridges, University of Canterbury, Queenstown, Nueva Zelanda.

Krawinkler, H. (1999), “Advancing performance-based earthquake engineering”, PEER Center Newsletter, Pacific Earthquake Engineering Research Center, Vol.2,No.1, enero.

Lobo Quintero, W. (1984), "Diseño de puentes bajo solicitaciones sísmicas", Memorias del IV Congreso Venezolano de Sismología e Ingeniería Sísmica, Barquisimeto, noviembre.

Lobo Quintero, W. (1996), "Diseño de edificaciones para dos estados límites", Seminario de Ingeniería Sísmica, Universidad Católica Andrés Bello, Caracas, Venezuela.

Lobo Quintero, W. (1997), "Puentes y tramos elevados: zonas críticas y medidas preventivas", Cap.11, Diseño sismorresistente, especificaciones y criterios empleados en Venezuela. Vol. XXXIII, Academia de Ciencias Físicas, Matemáticas y Naturales, Caracas, Venezuela.

Lobo Quintero, W. (1998), “Aislamiento sísmico de puentes”, Memorias, IV Jornadas CientíficoTécnicas, Facultad de Ingeniería, Universidad de los Andes, Mérida, Venezuela, noviembre.

Lobo Quintero, W. (1999), "Lineamientos para la actualización de las normas sismorresistentes de puentes, propuesta MTC-87", Memorias del VI Congreso Venezolano de Sismología e Ingeniería Sísmica, Mérida, Venezuela, mayo.

Loh, Ch-H., Wen-Yu, J., y Penzien, J. (1994), "Uniform-hazard response spectra-an alternative approach", Earthquake Engineering and Structural Dynamics, Vol. 23, pp. 433-445.

Maroney, B.H. y Chai, Y.H. (1994), "Bridge abutment stiffness and strength under earthquake loadings", Memorias, Second International Workshop on Seismic Design and Retrofitting of Reinforced Concrete Bridges, University of Canterbury, Queenstown, Nueva Zelanda.

Miranda, E. (1993a), "Site-dependent strength-reduction factors", ASCE Journal of Structural Engineering, Vol. 119, No. 12, diciembre.

Miranda, E. (1993b), "Evaluation of seismic design criteria for highway bridges”, Earthquake Spectra, Vol. 9, No. 2, pp. 233-250, mayo.

MTC-87 (1987), "Norma venezolana para el diseño sismorresistente de puentes", Ministerio de Transporte y Comunicaciones (propuesta de W. Lobo Quintero).

Nuti, C. y Ochat, E. (1994), "Comparison among seismic response of bridges with conventional and dissipative or elastic isolators designed to EC8", Memorias, Second International Workshop on Seismic Design and Retrofitting of Reinforced Concrete Bridges, University of Canterbury, Queenstown, Nueva Zelanda.

Okamoto, S. (1973), Introduction to earthquake engineering, John Wiley and Sons, Nueva York, y University of Tokyo Press.

Park, R. (1996), "New Zealand perspectives on seismic design of bridges", Memorias, XI World Conference on Eartquake. Engineering, Acapulco, México, Artículo No. 2111, CDROM.

Pincheira, J. A. (1993), "Design strategies for the seismic retrofit of reinforced concrete frames", Earthquake Spectra, Vol. 9, No. 4. 
Qi, X. y Moehle, J. P. (1991), "Displacement design approach for reinforced structures subjected to earthquakes", Reporte No UCB/EERC-91/02, Earthquake Engineering Research Center, University of California at Berkeley.

Ramírez, O. y Ayala, G. (1999a), "Método para el análisis sísmico no lineal de puentes. Modelo teórico y herramienta computacional”, Memorias del VI Congreso Venezolano de Sismología e Ingeniería Sísmica, Mérida, Venezuela, mayo.

Ramírez, O. y Ayala, G. (1999b), "Efecto de la variabilidad espacial de las ondas sísmicas en la respuesta no lineal de puentes", Memorias del VI Congreso Venezolano de Sismología e Ingeniería Sísmica, Mérida, Venezuela, mayo.

Ramírez, O. (1999), "Determinación de la vulnerabilidad sísmica de estructuras de puentes", Tesis Doctoral, División de Estudios de Posgrado de la Facultad de Ingeniería, UNAM, México, octubre.

Riddell, R., Hidalgo, P. y Cruz, E. (1989), "Response modification factors for earthquake resistant design of short period buildings", Earthquake Spectra, Vol. 5 No. 3.

Roberts, J. E. (1996), “U. S. perspectives on seismic design of bridges”, Memorias, XI World Conference on Eartquake. Engineering, Acapulco, México, Artículo No. 2109, CDROM

Saragoni, G. R., Lobos, C. y Gómez-Bernal, A. (1998), "Site and earthquake mechanism effect on design response spectra", Memorias, $11^{\text {th }}$ European Conference on Earthquake Engineering, Paris, Francia.

Sauter, F. (1998), "Medidas preventivas en el diseño sísmico de puentes", Memorias, $X$ SeminarioLatinoamericano de Ingeniería Sísmica, San José, Costa Rica, diciembre.

SEAOC (1995), "Vision 2000: Performance based seismic engineering of buildings", Structural Engineers Association of California, editor J. Soulkages, Sacramento, CA, abril.

Shibata, A. y Sozen, M. A. (1976), "Substitute-structure method for seismic design in RC", $A S C E$ Journal of Structural Engineering, Vol 102, No. 1, pp. 1-18.

Tena Colunga, A. (1997), "Evaluación de un método de diseño estático para el aislamiento sísmico de estructuras de la costa mexicana del Pacífico", Revista de Ingeniería Sísmica, SMIS, No. 57, pp. 1-34, septiembre-diciembre. 